\title{
Approche modèle pour une prise en charge globale des risques hydrologiques dans le bassin méditerranéen
}

\author{
Wahiba MENAD \\ Université Hassiba Benbouali Chlef - Laboratoire de Structures, Géotechniques et Risques \\ BP. 151 Hay Esalem 02000 Chlef Algérie - ( w.menad@ univ-chlef.dz ).
}

Manuscrit reçu le 31 mars 2017 et accepté le 5 novembre 2018

\section{Résumé}

Les risques d'ordre hydrométéorologiques sont les plus fréquents et les plus dévastateurs dans le monde. Le bassin méditerranéen n'échappe pas à cette règle, dont il abrite les formes les plus dangereuses. Néanmoins, une grande partie de son territoire, en particulier celui de la rive sud, n'a jamais été sujet d'une évaluation de risque hydrologique. Dans ce travail on a essayé de développer une méthode d'évaluation des risques hydrologiques notamment d'inondation, de crue et de ruissellement superficiel, causés par des événements météorologiques à caractère intense qui frappent le bassin méditerranéen. Il s'agit d'une méthode applicable sur l'ensemble du bassin, conçue sous forme d'une feuille de route dressant les principales étapes de celle-ci. Pour donner un certain aspect général à ce travail, il était nécessaire que les données utilisées dans les différentes étapes de l'étude soient disponibles et accessibles pour l'ensemble du bassin (gratuites ou à prix symbolique). Cette méthode est ainsi parfaitement transposable à d'autres territoires à leur image et encourant un même type de risque.

Mots clés : risque, crue, ruissellement superficiel, inondation, simulations numériques, évaluation, approche globaliste.

\begin{abstract}
Hydrometeorological risks are the most frequent and the most devastating in the world. The most dangerous forms exist in the Mediterranean Basin. Nevertheless, the hydrological risk was never estimated within the large part of the territory around the basin, in particular, the south shore. In this paper, we developed a method for evaluation of hydrological risks, specifiquely, flood, surface runoff and floods, caused by intense weather events. This method can be applied throughout the Mediterranean Basin and conceived as a roadmap outlining the main steps of the approach. To give a general aspect to this work, it was necessary, that data used in the various stages of the study are available and accessible (free or at a symbolic price) throughout the Mediterranean Basin. This method is perfectly transferable to other territories in their image and running the same type of risk.
\end{abstract}

Keywords : risk, flood, surface runoff, floods, holistic approach, assessment, modelling.

\section{Introduction}

Selon EM-DAT, les catastrophes d'ordre hydrologique représentent plus de $35 \%$ de l'ensemble des catastrophes naturelles qui ont frappé le bassin méditerranéen ces deux dernières décennies (1995-2015). Le climat méditerranéen s'inscrit dans un contexte particulièrement cyclogénétique, connu pour ses épisodes pluvieux de forte intensité et de courte durée. Les réponses hydrologiques déclenchées par ce type d'événement météorologique sont sou- 
vent efficaces (réponse rapide, forts débits de pointe) et aux conséquences graves voire destructives.

Devant l'ampleur croissante du risque hydrométéorologique, les scientifiques, les politiciens et les gestionnaires en ont fait une priorité. Et le grand nombre de travaux réalisés au cours de ces dernières années dans chacune de ces disciplines en témoigne.

On compte sur les deux précédentes décennies un nombre important d'études qui se sont penchées sur l'analyse des processus atmosphériques générateurs de pluies intenses ainsi que sur leurs relations avec les facteurs environnementaux (Lasat et Rodriguez., 1997 ; Homar et al., 2003 ; Lebeaupin et al., 2006 ; Ducrocq et al., 2007, Argence, 2008, El Saidi et al., 2016). Les connaissances en ce domaine ont connu de véritables avancées, en particulier après la mise en place des réseaux de radar qui permettent de suivre et fournir des informations en temps réel sur l'évolution des configurations atmosphériques. Dans le bassin méditerranéen, le projet Arc Méditerranéen (1999-2004) a donné un vrai coup de pousse en ce qui concerne la généralisation des réseaux de prévision (Delrieu, 2005). On trouve aussi le ClimChAlp, programme européen dans lequel la problématique du changement climatique est étudiée afin de proposer des stratégies de développement durable adaptées aux conditions de la région.

La maîtrise de l'aléa hydrologique a également connu de grandes avancées. Un aléa classiquement caractérisé par : la hauteur et la durée de submersion, la vitesse des écoulements, et le débit (Torterotot, 1993 ; Barozet et al. 1994). La modélisation hydrologique (modèles empiriques globaux, modèles conceptuels, modèles à base physique) et, en particulier, la modélisation numérique dont les bases ont été jetées par M.B Abbott en 1991, représente une entreprise moderne qui a et qui continue à révolutionner le domaine de l'hydrologie ; grâce à elle, de nombreuses limites classiques ont pu être surmontées (Ghidaglia et Rittaud, 2004). Les modèles numériques qui existent actuellement sont dotés d'une performance acceptable et d'une utilisation conviviale mais sans pour autant arriver à l'objectif espéré, qui consiste à remplacer les experts par un modèle numérique facilement manipulable, dans lequel toutes les connaissances d'un expert physique sont incorporées (Harvey, 2002; Ambroise, 2012). Néanmoins, leur conceptualisation, adaptation et performance sont en permanente évolution.

L'évaluation de la vulnérabilité repose sur une analyse fine des enjeux (population, mode d'occupation et d'usage des sols) exposés à l'aléa. Il existe principalement trois grandes approches appliquées dans l'analyse et l'évaluation de la vulnérabilité : 1) analyse coûtsbénéfices $(\mathrm{ACB})$ : cette approche est très largement admise dans de nombreux pays tels que la France (Hubert et Bruno, 1999), les États-Unis (USWRC, 1983), l'Angleterre (PenningRowsell, 1999), les Pays-Bas (Herman et al., 1999). Selon Gaber (2007), une ACB consiste à « comparer divers scénarios d'action entre eux ou par rapport à la situation de référence sur les base de coûts actualisés dans le temps et des bénéfices attendus actualisés sur la même période »;2) approche zonale : elle se base sur un partage du territoire en plusieurs unités spatiales homogènes (maximum d'homogénéité intrinsèque). À chacune de ces unités est assignée une densité moyenne d'enjeux et de là un taux d'endommagement moyen pour chacune d'entre elles. L'application de l'approche zonale en milieu urbain reste une tâche difficile (Sageri, 1987) ; 3) approche par agents : il s'agit d'établir dans un premier temps une typologie très fine des enjeux présents sur un territoire donné puis, dans un second temps, d'associer un taux d'endommagement à chaque type d'enjeux. Ce qui pose problème dans cette approche est la multitude de typologies d'agents présents dans la littérature, car selon la formation de l'auteur (politicien, urbaniste, économe ...) les classes d'enjeux formées diffèrent (Devaux-Ros, 1999 ; Hubert et Ledoux, 1999 ; Barroca et al., 2008).

Le but de ce travail est de proposer une méthode de prise en charge globale des territoires, non étudiés auparavant, sujettes à des épisodes météorologique à caractère intense qui 
frappent le bassin méditerranéen. Une méthode conçue pour être parfaitement transposable sur l'ensemble des territoires à leur image.

\section{Méthodologie}

Dans le bassin méditerranéen, en particulier sur sa rive sud, le risque hydrométéorologique reste méconnu, aucun plan de prévention n'ayant été établi. Concernant cette problématique, tout reste à faire : identification, analyse et gestion des zones à risque.

Dans ce travail on a essayé de développer une méthode de prise en charge globale des risques hydrologiques notamment d'inondation, de crue et de ruissellement superficiel, déclenchés par des événements météorologique à caractère intense qui frappent des zones du bassin non étudiées auparavant. Il s'agit d'une méthode applicable sur l'ensemble du bassin méditerranéen, conçue sous forme d'une feuille de route dressant les principales étapes de celle-ci. Pour donner un certain aspect général à ce travail, il était nécessaire que les données utilisées dans les différentes étapes de l'étude soient disponibles et accessibles (gratuites ou à prix symbolique) pour l'ensemble du bassin. Cette méthode est ainsi parfaitement transposable à d'autres territoires à leur image et encourant un même type de risque.

Pour y parvenir, cette démarche sera menée en plusieurs étapes (Fig. 1) :

- Inspection du terrain, via des enquêtes, pour une localisation spatiale et temporelle (saison, mois) de zones à risque hydrologique ;

- Collecte et/ou gènes des données nécessaires pour mener à bien ce genre de démarche globale d'évaluation du risque;

- Caractérisation et évaluation des niveaux de risques hydrométéorologique encourus avec une approche thématique ;

- Pour les zones à hydrosystème et/ou à enjeux complexes, l'analyse va être poussée plus loin avec des approches hydrosystmiques basées sur des outils de simulation numérique, qui permettent de considérer l'hydrosystème dans sa globalité ;

- L'ensemble des résultats obtenus vont être complétés (si besoin) par une analyse systémique, hiérarchisant les facteurs prédisposants, déclenchants et aggravants, non supportés par les outils numérique de simulation ;

- Edition d'un support cartographique : à la lumière de l'ensemble des analyses (thématique et systémique), des calculs et des simulations réalisés, un support cartographique illustrant le niveau du risque hydrométéorologique est édité.

Cette démarche a été testée lors d'un travail de thèse réalisé sur le Massif de Bouzaréah, qui se situe dans la partie ouest du Grand Alger (Algérie ; Fig. 2). Le Massif de Bouzaréah, d'un point de vue hydrologique, est formé de trois bassins-versants, à savoir: le Beni Messous, l'Oued Koriche et un ensemble de sous-bassins versants Côtiers. Pour mieux expliquer certains points de la démarche, on va s'appuyer sur des illustrations produites lors de ce travail. 
Bulletin de la Société Royale des Sciences de Liège, Vol. 88, Article, 2019, p. 1 - 21

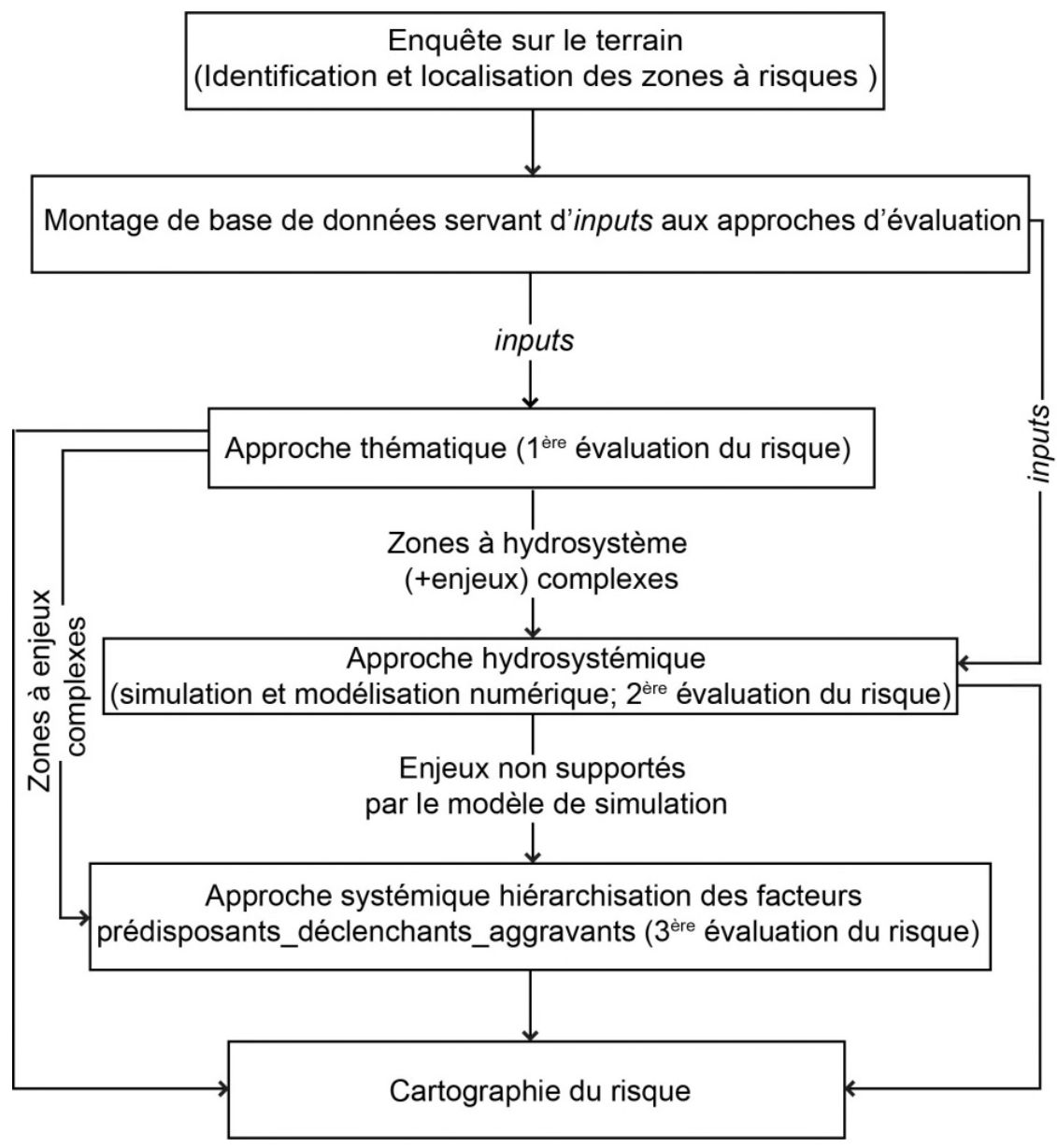

Fig. 1 : Méthodologie de prise en charge globale du risque d'ordre hydrologique

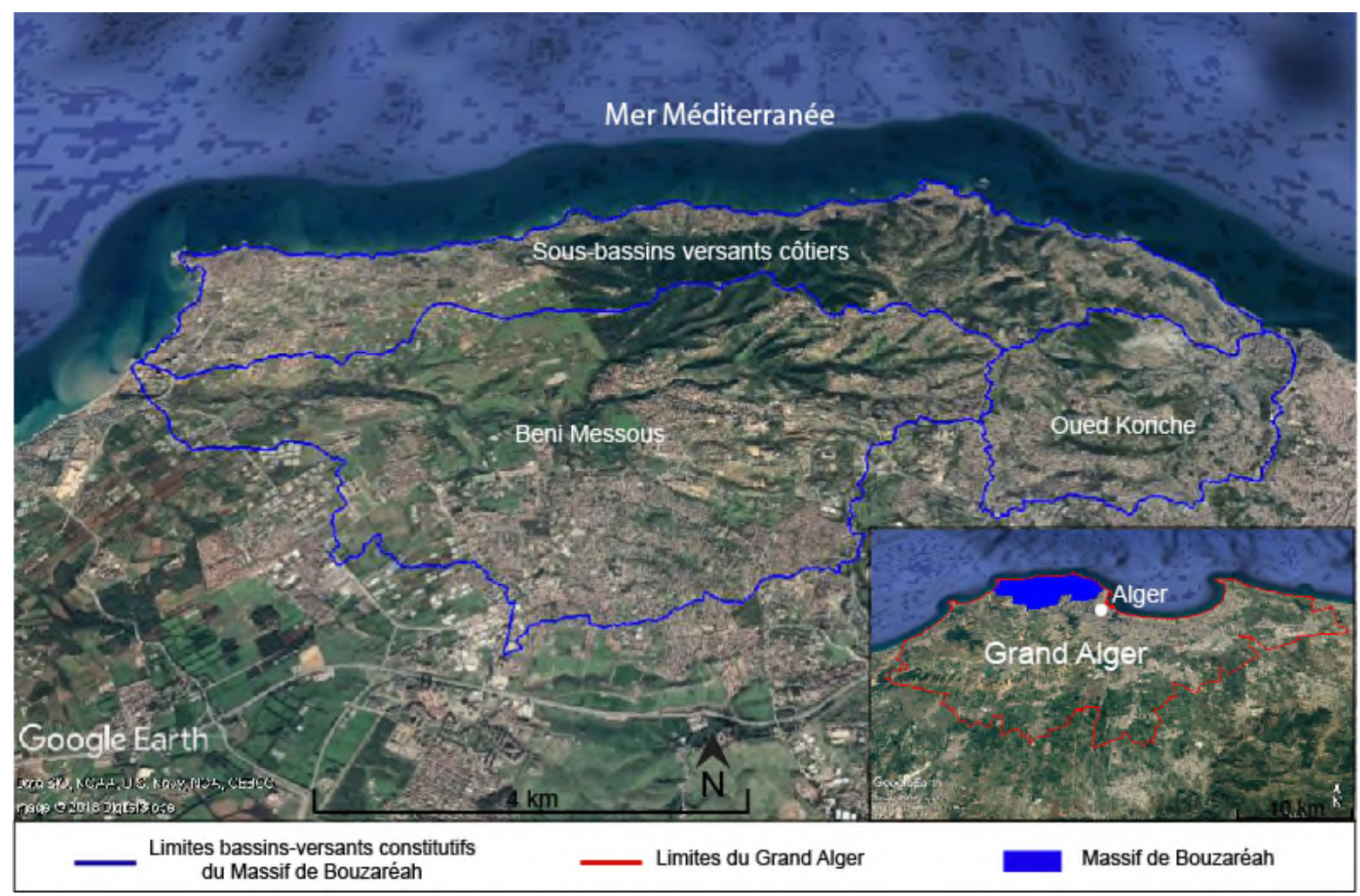

Fig. 2 : Carte de localisation du Massif de Bouzaréah 


\section{Localisation des zones à risques et détermination de leur extension spatio-temporelle}

Partant du fait que le bassin méditerranéen présente un aléa météorologique (événements cyclogénétiques) favorable au déclenchement de réponses hydrologiques à risque, ainsi on suppose que l'ensemble du bassin méditerranéen est exposé à un probable risque d'ordre hydrométéorologique. À ce niveau, il s'agit d'identifier les zones réellement exposées, le type de risque existant (crue, inondation ou ruissellement superficiel), et de définir, dans la mesure de possible, son extension spatio-temporelle.

Pour mener à bien et avec efficacité cette étape, il est conseillé de procéder via une enquête conçue sur des bases hydrologiques. Cette enquête permettra une première exploration qualitative, dont on va identifier le type de risque encouru (inondation, crue, ruissellement superficiel), recenser les événements du passé et définir l'événement de référence, évaluer son extension spatio-temporelle, leur période d'apparition (saison, mois), relever certaines caractéristiques ou traits physiques du terrain (système de pente, nature des formations superficielles, leur perméabilité, régime hydrologique, formes d'érosion,...), relever les activités anthropiques qui peuvent probablement aggravées le risque existant (mode d'occupation du sol, artificialisation du réseau hydrographique, type et état du réseau d'assainissement ...). Il s'agit uniquement d'une évaluation qualitative, basée sur des observations faites directement sur le terrain. Pour consulter des modèles de ce genre d'enquête on renvoi le lecteur vers le travail de Menad (2005) et celui de Douba et Hamidi-Boudjalthia (2016).

Le but principal de ces enquêtes étant d'identifier les zones présentant un réel risque hydrologique, d'observer l'environnement dans lequel il évolue. Cette première qualification à un intérêt capital pour la suite du travail, et notamment lors du montage de la base de données. Cette étape permet un gain de temps et d'argent considérable ; traditionnellement on commence avec une démarche thématique (cf. infra) où on est amené à monter une base de données couvrant tout le territoire à étudier ; cela implique également d'analyser et d'évaluer les paramètres physiques, hydrologiques et sociaux sur l'ensemble de ce dernier. Alors qu'en commençant par localiser les zones réellement touchées, cela réduira significativement la charge du travail.

\section{Montage de la base de données}

Pour pouvoir mener à bien une analyse globale du risque hydrologique (volets aléa et vulnérabilité), une base de données importante doit être établie en amont.

\section{III.1. Données nécessaires à l'analyse météorologique}

Souvent les données météorologiques et climatologiques sont dispersées entre plusieurs services. De ce fait, un travail de collecte de donnée minutieux doit être effectué :

- Récupérer les bases de données existantes au niveau des services concernés (stations météorologiques, services d'étude climatologique, services d'hydrologie, collectivités locales,...), exploiter leur travaux tels : rapports et fiches techniques publiés ; étude d'impact des différents projets, etc ...;

- Exploiter les travaux scientifiques réalisés sur la région à considérer (article, mémoire de fin d'étude, rapport de stage, etc ...);

- Exploiter des archives telles que : les articles de presse, les écrits littéraires, les courriers de communication et les documents officiels des collectivités locales ;

- Les enquêtes de terrain réalisées auparavant (cf. supra) vont également nous aider à 
repérer les événements remarquables du passé.

Dans un grand nombre de pays sous-développés les enregistrements météorologiques à échelle spatio-temporelle fine sont défaillants, du fait d'un réseau de stations de mesures dispersé, induisant un manque de couverture spatiale. Les relevés sont également réalisés à échelle temporelle de quelques heures et rarement de l'ordre de minutes. En réalité cette problématique se pose également dans les pays de la rive nord, car les événements cyclogénétique méditerranéens, à l'origine de comportements hydrologiques à risque, sont très localisés spatialement, les chances que l'épicentre d'un événement pluvieux coïncide avec un point de mesure restent faibles. Ainsi, la reproduction spatio-temporelle fine des événements météorologique reste une étape compliquée, mais nécessaire pour reproduire le plus fidèlement possible la réponse hydrologique instantanée du bassin-versant. Pour remédier à cette lacune récurrente on fait recourt à un couplage d'informations fournies par stations de mesures hydrologiques locales, les mesures des stations avoisinantes (hyétogramme) ainsi que les échosradars (si disponibles).

Les enregistrements radar (échos radars) : quand ils sont disponibles ils présentent un document de grande valeur, ils permettent de confirmer les enregistrements au sol et dans certains cas de localiser l'épicentre de l'événement. Les enregistrements radar permettent également la détection, en amont, des configurations atmosphériques à risque, et donc prévenir leur arrivée, estimer leur probable intensité, et suivre leur évolution spatio-temporelle.

Le témoignage de la population peut, dans certains cas où les mesures sont sérieusement défaillantes, être utilisé pour affiner la répartition temporelle (replacer dans le temps le ou les pics d'intensité) voire spatiale de l'épisode pluvieux.

\section{III.1.1. Données nécessaires à l'analyse de l'hydrosystème}

L'étude du comportement hydrologique est communément menée à l'échelle du bassinversant via un nombre de paramètres influençant directement ou indirectement ce dernier (cf. infra). Avec l'arrivée de l'outil informatique (simulation ou modélisation numérique) il est devenu possible d'étudier la réponse hydrologique instantanée à tout point du bassin-versant. De plus en plus des études démontrent l'utilité d'aller au-delà de l'unité du bassin-versant, et d'étudier la réponse hydrologique au niveau du sous-bassin versant voire à tout point de ce dernier (Douvinet et al, 2008 ; Menad, 2012). En réalité, certains comportements locaux à risques (anomalies) sont masqués à l'échelle de la réponse globale.

Ainsi, pour mener une analyse fine de l'hydrosystème un nombre de données d'entrée important sont à préparer en amont :

A. Topographie: les données topographiques du terrain sont incontournables dans ce genre d'étude. Elles sont souvent représentées sous forme d'un modèle numérique de terrain (MNT).

Il existe des bases de données mondiales où les MNT sont disponibles gratuitement telles les bases ASTER, MNT SRTM. La résolution la plus fine proposée gratuitement par ces bases de données est généralement de l'ordre de $30 \mathrm{~m}$. Pour une analyse plus poussée de l'hydrosystème, et selon qu'on est en plaine ou en zone de pente, le montage d'un MNT plus fin est souvent indispensable. Le niveau de résolution requis est donc relatif au résultat recherché et à la nature de terrain étudié, les zones en pente nécessitent un MNT moins fin qu'une zone en faible pente. Pour reproduire des MNT à pas plus fin, on fait recours aux outils de SIG, qui permettent de digitaliser les courbes de niveaux à partir de fonds de cartes topographiques. Ce dernier est un document facilement accessible et souvent disponible à prix symboliques. 
Le MNT est une donnée d'entrée (input) importante pour toute opération numérique de simulation ou de modélisation hydrologique. C'est également un document de base pour l'analyse des paramètres hydrologiques, et pour dériver de nombreuses informations telles que : la délimitation des unités hydrographiques (bassin-versant, sous-bassin-versant), calculs des données géométriques ; reproduire le réseau hydrographique et déterminer sa typologie et sa hiérarchisation, caractériser le relief et le système de pentes.

B. Formations superficielles et dynamique géomorphologique : les formations superficielles déterminent en grande partie la nature de la réponse hydrologique. Le problème qui se pose concernant les cartes de formations superficielles est celui de leur actualisation. Les formations superficielles d'origine anthropique (remblais, débris d'origine divers, ...) manifestent une évolution spatio-temporelle souvent rapide et anarchique par endroits, en comparaison avec celles conditionnées par des facteurs d'ordre physique (relief, structure, climat, hydrologie). D'où la nécessité de réaliser un travail d'actualisation avant de commencer l'analyse du risque.

Dans le cas où la carte de formations superficielles est indisponible, son établissement s'impose. Pour y parvenir, il faut croiser un grand nombre d'informations, telles que : les cartes géologiques, topographique, agricoles et pédologiques, coefficients d'infiltrations, les rapports d'études géotechniques, sondages du sous-sol de l'ordre de 10 à $15 \mathrm{~m}$ de profondeur, mesures de résistance de terrains (mesures de pénétromètres statique), cisaillement des terrains (sismique réflexion), etc. Ce genre d'informations présentent l'avantage d'être largement disponibles, car elles sont fournies par les études d'impacts réalisées en amont de projets de natures diverses (installation de zones urbaines, industrielles, d'infrastructures, ...). Les outils de télédétections sont de plus en plus utilisés dans les reproductions lithologiques. L'exploitation de travaux scientifiques est également fortement recommandée dans cette quête de la donnée (thèses et mémoires de fin d'étude, rapports de stages, publications scientifiques, etc.).

\section{III.1.2. Données nécessaires à l'analyse du contexte sociétal}

Pour évaluer le volet vulnérabilité du risque hydrologique, et mener une analyse fine des enjeux exposés à l'aléa, un certain nombre de données doit être collecté.

Souvent dans l'étude des risques hydrologiques, l'analyse de la vulnérabilité se limite au sens physique du terme, faisant uniquement référence à l'exposition du territoire physique à un aléa. L'analyse porte rarement sur la sinistralité des crues, sur les impacts économiques indirects, sur les aspects sociaux ou encore psychologiques. Ces dernières engagent un important travail d'équipe interdisciplinaire, il s'agit de démarches gourmandes en argents et en main-d'œuvre. Ces aspects présentent également l'inconvénient de fournir des évaluations à caractère qualitatif telle l'évaluation psychologique des sinistrés par exemple. La confrontation des évaluations qualitatives et celles quantitatives des deux volets du risque (aléa, vulnérabilité) reste très difficile à réaliser.

Pour la méthode développée dans ce travail la vulnérabilité sera qualifiée à travers les aspects suivants :

A. Répartition spatio-temporelle des enjeux exposés à l'aléa: la répartition spatio-temporelle des enjeux sur le terrain peut être reproduite à travers l'établissement de cartes d'occupation du sol. La réalisation d'une étude diachronique de l'occupation du sol (évolution urbaine et modifications spatio-temporelle de l'environnement en général) peut s'avérer utile pour pousser plus loin les analyses hydrologiques et prévoir l'impact des futures évolutions. D'un autre côté, ces cartes renseignent directement le coefficient de ruissellement ou le refus d'infiltration qui est une donnée d'entrée nécessaire pour mener les modélisations et/ou les 
simulations numériques.

Pour produire des cartes d'occupation du sol, avec des moyens financés réduits on propose ici deux méthodes (Menad, 2012) :

A.1. Méthode de photo-interprétation thématique : en exploitant, avec des outils de SIG, les fonds de cartes topographique, des orthophotos, des images de Google Earth, des plans cadastraux, etc ; les principaux objets constructifs de l'environnement sont décrits, puis des cartes d'occupation du sol sont éditées.

A.2. Traitement numérique des images satellites : il existe de nombreuses bases de données mondiales qui peuvent fournir gratuitement des images satellites, telles les images LANDSAT connues pour être de bons supports à l'analyse thématique et présentant l'avantage d'une acquisition à faible coût (gratuites à partir de $30 \mathrm{~m}$ de résolution). Elles donnent accès à une évaluation, qualitative et quantitative, fiable des principaux types d'occupation du sol existant sur le terrain (Menad, 2012).

B. Collecte des textes législatifs traitants la problématique des risques hydrologique ou encore des risques majeurs d'une manière générale. Le but ici, c'est d'être en accord avec la législation au moment de proposer des mesures de prévention du risque (but ultime d'une étude du risque).

\section{IV. Évaluation du risque avec une démarche thématique (1 ${ }^{\text {ére }}$ évaluation)}

Il s'agit d'une démarche d'analyse classique ou thématique, ou chacun des facteurs du milieu impliqués dans la genèse et le développement du risque est étudié plus au moins indépendamment de l'autre. Une démarche idéalement applicable sur des terrains qui font l'objet d'une première évaluation du risque. Pour des terrains à faibles enjeux ou à hydrosystème simple la méthode thématique peut déboucher sur une cartographie du risque. Pour des zones à enjeux et/ou à hydrosystème complexes, l'approche thématique s'avère limitée et l'analyse doit être poussée plus loin en utilisant des démarches hydrosystémique ou systémique, plus gourmandes en données et impliquant du matériels et d'outils d'analyses plus coûteux.

Pour pouvoir mener à bien une démarche thématique certains paramètre doivent être étudiés, à savoir :

\section{IV.1. Étude du contexte physique (volet aléa)}

Les principales caractéristiques physiques de la région étudiée seront évalués à travers :

\section{IV.1.1. Facteurs météorologiques voire climatiques influençant l'aléa hydrologique}

Il s'agit du premier facteur déclencheur de la réponse hydrologique. En se servant des données collectées durant la deuxième étape de notre démarche, le contexte climatique régional puis local seront définis, à travers une : caractérisation des conditions climatiques générales (types du climat, caractéristiques des précipitation annuelles, mensuelles et journalières moyennes, ...), localisation spatiale et temporelle (saison) des événements météorologiques de forte intensité (i.e. pluie est considéré comme intense quand un cumul pluvieux en $24 \mathrm{~h}$ égal ou dépasse celui reçu habituellement en un mois), calculs des périodes de retours (décennales, cinquantennales, centennales, ...), détermination des mécanismes atmosphériques intervenants dans le déclenchement des réponse à risque et définir le niveau de fiabilité de leurs prévision à l'échelle temporelle (semaines, jours, heures) ainsi qu'à l'échelle spatiale (microéchelle, méso-échelle, macro-échelle). 
Certains épisodes pluvieux résultent de configurations atmosphériques typiques et tout à fait prévisibles à grande échelle (Chaboureau et al., 2001); en revanche, leur comportement à micro-échelle (échelle convective) est conditionné par un complexe interactif de plusieurs processus qui, à ce jour, restent mal connus. D'autres événements peuvent résulter d'orages très localisés qu'ils n'ont pas de précurseurs caractéristiques à grande échelle.

En Méditerranée, les mécanismes intervenant dans la genèse des événements intenses, peuvent être classés, selon leur échelle temporelle (de quelques heures à plusieurs jours), leur échelle spatiale (dizaines à plusieurs centaines de kilomètres) et les conditions initiales régnant sur le milieu au moment de leurs genèses, en trois catégories, à savoir : les cyclogenèse, systèmes perturbés provenant de l'Atlantique Nord, systèmes convectifs de méso-échelle et orages orographiques. La compréhension des mécanismes de formation et d'évolution de ces événements intenses reste délicate, particulièrement à méso- et micro-échelle, en raison de la multitude des processus impliqués dans leurs genèses, ainsi que leurs chevauchements et interactions souvent non linéaires (Maheras et al., 2001, Argence, 2008).

\section{IV.1.2. Substrat, formations superficielles et dynamiques géomorphologiques}

À ce niveau la nature des formations superficielles et les mécanismes de leur mise en place seront déterminés, ainsi que les facteurs de contrôlant l'évolution géomorphologique. Ces facteurs sont d'ordre physique (relief, structure, climat, hydrologie) et/ou anthropique (défrichement, pratiques agricoles mal adaptées, aménagement du territoire en milieu urbain). On détermine également : les coefficients de perméabilité ou de refus d'infiltration ; sensibilité aux phénomènes d'érosion, quantité et la nature de la charge solide qui peut être fournie aux écoulements ; ainsi que le mode de genèse des écoulements de surface (écoulement de surface de type hortonien, l'aptitude à former des croûtes de battances, ruissellement sur surface saturée).

\section{IV.1.3. Délimiter et caractériser les unités hydrographiques}

Les caractéristiques physiographiques d'un bassin-versant jouent un rôle déterminant dans son comportement hydrologique. L'ensemble des facteurs impliqués dans la caractérisation des unités hydrologiques sont facilement et rapidement déduits à partir d'un MNT manipulé avec un SIG, ou encore avec des méthodes classiques à partir d'un support topographique.

- Surface du bassin-versant: est présentée souvent en $\mathrm{km}^{2}$. La surface d'un bassinversant donne une première idée concernant les volumes d'eau susceptibles d'être générés.

- Forme : la forme d'un bassin-versant se décrit à partir de la forme de son périmètre. Elle est souvent utilisée pour comparer entre les bassins versants. Selon qu'il s'agit d'une forme allongée ou circulaire, l'allure de l'hydrogramme de crue calculé à l'exutoire du bassin-versant sera très différente.

- Relief et pente : le relief est l'un des facteurs clés contrôlant la réponse hydrologique d'un bassin-versant, les pentes influencent à la fois les vitesses et les puissances spécifiques des écoulements ainsi que le temps de la réponse hydrologique. Ils peuvent être qualifiés avec plusieurs méthodes telles l'établissement de la courbe hypsométrique ou le calcul du rectangle équivalent. La cartographie du système de pente est également une bonne technique d'analyse, elle fournit à la fois des informations d'ordre qualitatives (répartition spatiale des pentes : zone à forte pente, zones à pente moyenne, zone plane, etc) et quantitatives (degré de pente).

- Réseau hydrographique : il représente très probablement l'élément de caractérisation le plus important de l'analyse hydrologique d'un bassin-versant. Il peut être caractérisé par 
une méthode descriptive d'ordre qualitative dite «topologique », avec laquelle le réseau hydrographique sera hiérarchisé et classifié (Shreve, 1966; Howard, 1967). La méthode d'hiérarchisation de Strahler, dans le cadre de notre étude, est peut être la meilleure, elle met en évidence la magnitude du réseau hydrographique montrant ainsi l'importance des segments élémentaires. Le réseau hydrographique est également caractérisé à travers : le calcul du rapport de confluence qui relève la présence (ou pas) de probables zones à risque de débordement ; la longueur moyenne des drains ; la densité de drainage et l'établissement d'un modèle de drainage, hydrogramme unitaire.

Pour mener à bien une analyse hydrologique il est également important d'identifier les types de régimes d'écoulement existants sur le terrain à considérer (fleuve, rivière, torrent, oued, écoulement diffus, ...).

L'ensemble de ces informations sont indispensables pour comprendre la dynamique des eaux superficielles et déterminer le temps de la réponse hydrologique des bassins versants, voire même établir une première cartographie de l'aléa (zones de forte confluence par exemple).

\section{IV.2. Étude du contexte sociétal (volet vulnérabilité)}

L'anthropisation du milieu physique peut changer radicalement le système hydrologique. En se servant des cartes d'occupations du sol réalisées auparavant, et sur lesquelles les principaux types de couvertures de sol sont décrits (zones urbaines, zones industrielles, parcelles agricoles, forêts, sol nu, ...), des carte couplées avec celles reproduisant la répartition spatiale de la population (densité de la population); un premier document cartographique, présentant les différents niveaux de vulnérabilité, peut être établi.

L'expérience montre que la prise en compte des enjeux mobiles dans l'évaluation du niveau de vulnérabilité et le développement des stratégies de gestions, s'avère très importante. Ainsi, pour un même milieu physique, et selon la dynamique de ces enjeux mobiles, plusieurs cartes de vulnérabilités peuvent être produites (carte de vulnérabilité dynamique). Par exemple on produit une carte de vulnérabilité aux heures de pointes, où les routes sont chargées; une autre carte va représenter la répartition des enjeux mobiles aux périodes où les commerces, les lieux de travail et les écoles sont très fréquentés, en période nocturne ce sont généralement les zones d'habitation à prédominance résidentielle qui sont les plus vulnérables.

Pour mieux comprendre l'intérêt à considérer des enjeux mobiles on prend l'exemple des crues catastrophiques qui ont frappées, en 2001, le bassin-versant de l'Oued Koriche (partie ouest du Grand Alger de $10 \mathrm{~km}^{2}$ de surface, de forme trapue asymétrique, à pentes fortes voire raides par endroit, réseau hydrographique de type dendritique dense à caractère torrentiel en aval ; Menad, 2012) et qui ont causé près de 1000 morts. Cet événement aurait certainement était moins catastrophique s'il n'avait pas frappé le matin aux heures de pointes. Le plus grand nombre de pertes humaines a été enregistré sur la route principale qui longe ce bassin-versant (i.e. cette route n'est autre que le lit mineur du bassin en question) ou encore en extrême aval de ce dernier où on trouve un concentré d'habitations, de commerces, de marchés et d'administrations. Un aléa de même hauteur frappant la nuit aurait causé beaucoup moins de perte en vies humaines. Ainsi les informations concernant les enjeux mobiles sont très importantes lors de l'élaboration et l'application d'une politique de gestion et de prévention du risque hydrologique en zones urbaines.

La restitution du cadre politique et gestionnaire, dans lequel le risque est considéré est également une démarche importante à entreprendre, elle s'avère décisive lors du développement des mesures de préventions, ça permit de proposer des mesures en adéquation avec l'environnement dans lequel le risque évolue. 


\section{IV.3. Apports de la démarche thématique}

À la fin de l'analyse thématique, de contexte physique, sociétal et le climat politique et gestionnaire dans lequel le risque hydrologique évolue, auront fait l'objet d'une première évaluation.

Les résultats de l'analyse du contexte physique sont présentés via un support cartographique sur lequel on superpose des cartes de formations superficielles, d'occupation du sol et donc coefficient de perméabilité, carte de pente, réseau hydrographique, zones de confluences, et toutes autres informations reliées au contexte physique et influençant la réponse hydrologique. Cette superposition de l'information va faire ressortir les différents niveaux de l'aléa (aléa hydrologique fort, moyen, faible). L'analyse du contexte sociétal permettra, elle aussi, de produire une carte avec les différents niveaux de vulnérabilité présents, ou encore plusieurs cartes traitant les enjeux mobiles. La confrontation des deux cartes (aléa, vulnérabilité) permettra de cartographier les différents niveaux du risque.

\section{Approche hydrosystémique (simulation et modélisation numérique)}

Les territoires révélés, par la méthode thématique, être à haut risque et/ou à hydrosystème complexe, doivent être réévalués par une approche qui considère l'hydrosystème dans sa globalité.

En approche thématique on procède avec des méthodes d'analyses classiques, ces dernières présentent l'inconvénient d'être sectorielles, où les paramètres sont étudiés séparément sans pouvoir être mis directement en interaction. Néanmoins, les différents paramètres de l'hydrosystème sont liés par des mécanismes complexes gérés par des interactions non linéaires, ainsi les variables environnementales (météorologie, physiographie, formations superficielles et facteurs anthropique) ne peuvent être considérées séparément. D'où tout l'intérêt de recourir à des méthodes hydrosystémiques qui permettent de mettre simultanément en jeu l'ensemble de ces paramètres. Il s'agit de comprendre le fonctionnement de l'hydrosystème au-delà de la perception du bassin-versant comme étant une unité hydrologique homogène. Partant du constat que certains comportements locaux peuvent être masqués dans la réponse globale, un nombre de travaux souligne la nécessité de revisiter le concept du bassin-versant (Douvinet, 2010 ; Menad, 2012). Pour y parvenir, on fait, de plus en plus, recoure aux outils de simulation numérique.

Les modèles de simulation ou de modélisation numériques sont devenus des outils incontournables, dont la conceptualisation, adaptation et performance sont en permanente évolution. Selon les caractéristiques du territoire, du phénomène hydrologique et du type d'information recherché, les outils numériques vont varier entre modèles de simulation globaux et modèles de modélisation spécialisés voire très spécialisés. Ces derniers permettent d'effectuer des analyses très poussées et raffinées afin de traiter une problématique localisée dans l'espace et dans le temps, et viennent souvent compléter des outputs fournis au préalable par des modèles globaux, ou encore par une démarche thématique

À ce niveau, On peut citer l'un des outils de simulation numérique développé en géographie, à savoir l'automate cellulaire RuiCells, qui a été utilisé dans deux travaux de thèse (Douvinet, 2010 ; Menad, 2012). RuiCells a fait preuve d'une grande utilité pour mener des travaux de géomorphologie et d'hydrologie aux niveaux des bassins-versants. Sa structure et son principe de fonctionnement permettent d'étudier l'influence de l'organisation morphologique du bassin-versant, entendue comme étant l'association des effets joués par la forme du bassin, la hiérarchie des réseaux hydrographiques et le système de pentes, avec les effets liés à l'agencement spatial des surfaces ruisselantes ou infiltrantes (Delahaye, 2002 ; Langlois et 
Delahaye, 2002 ; Douvinet et al., 2008 ; Menad et al., 2012). D'autres paramètres de l'hydrosystème, tel les modes d'occupation du sol et les épisodes pluvieux du passé (hyétogramme), peuvent être également implémentés.

Pour mener à bien une analyse globale de l'hydrosystème, on propose de la réaliser en deux étapes :

\section{V.1. Analyse de l'efficacité morphologique et de l'évolution spatio-temporelle de la ré- ponse hydrologique}

L'efficacité morphologique doit être étudiée en tout point de l'espace à travers une mise en jeu progressive des différentes composantes du système hydromorphologique (forme, efficacité structurale du réseau d'écoulement, système de pentes), à travers cela, de nombreuses informations vont être déduites, dont les principales sont :

- Identification de la nature des mécanismes qui gèrent les interactions entre les composantes morphologiques ainsi que leur inscription spatiale et temporelle dans le bassin-versant (emboîtement des sous-bassins versants et les distances qui leurs séparent de l'exutoire final);

- Quantification de l'effet exercé par chacune des composantes morphologiques sur la réponse hydrologique théorique, à travers : 1) des indices morphosynthétiques, dont on peut produire un distançogramme (Fig.3), une carte d'écoulement de surface (Fig.4) et un surfaçogramme (Fig.5 ; A). Il s'agit, de simulations dans un espace 2D, dans laquelle on fait transiter respectivement un cumul du nombre de cellules équidistantes et des cumuls de surface de cellules équidistantes ; 2) d'indices physiographiques, tel le $I E$ : la racine carrée de la surface globale du bassin-versant est rapportée au pic de la réponse surfacique Qmax le tout multiplier par 100. Un IE équivalant à 100 renseigne sur une efficacité structurelle maximale du réseau, où la bande des cellules équidistantes ${ }^{1}$ coïncide avec le diamètre moyen du bassin. Le plus grand avantage que présentent ce type d'indices est qu'il compare des fonctionnalités, ce qui permet de s'affranchir de la barrière de la taille souvent rencontrée dans une démarche comparative entre des bassins-versants de tailles distinctes.

- Pour étudier l'évolution de la réponse hydrologique (estimation des débits et des volumes écoulés en tout point de l'espace) et son efficacité dans le système morphologique dans lequel elle se développe, on doit procéder par une simulation 3D. Cette simulation combine les principaux paramètres de l'hydrosystème à savoir : la forme, la structure du réseau, le système de pentes, ainsi qu'un l'hyétogramme (théorique ou d'événements réels). En sorties on obtient une cartographie de l'évolution spatio-temporelle de la réponse hydrologique ainsi que le morphogramme correspondant (Fig.5 ; B), ce qui fait ressortir certains comportements locaux à risque (anomalies) masqués à l'échelle globale (Douvinet, 2010 ; Menad, 2012).

\footnotetext{
${ }^{1}$ Bandes de cellules situées à égale distance de l'exutoire.
} 
Bulletin de la Société Royale des Sciences de Liège, Vol. 88, Article, 2019, p. 1 - 21
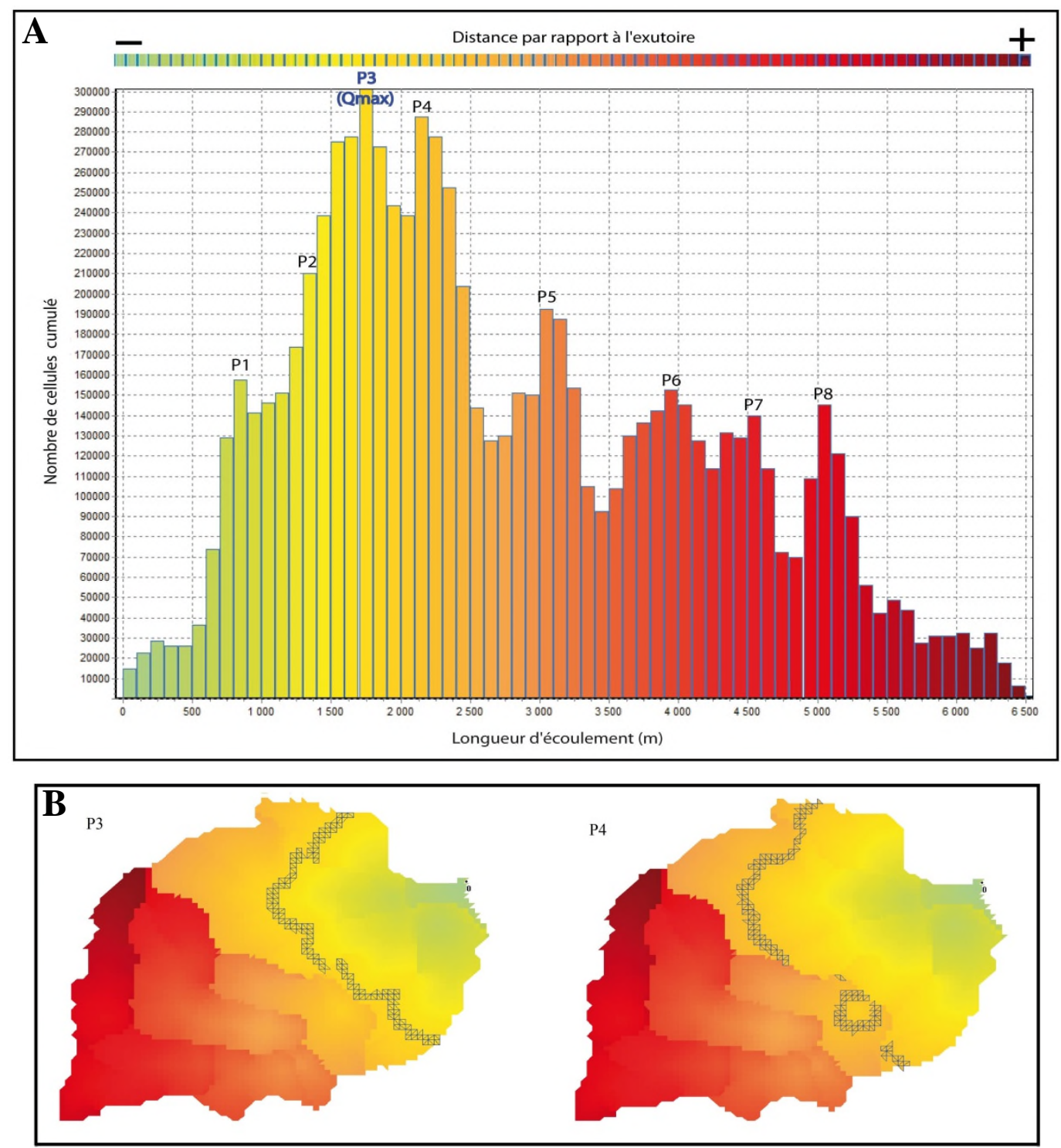

Fig.3 : A : Distançogramme. B : Cartographie des cellules à équidistance de l'exutoire du bassin versant de l'Oued Koriche, (modifier selon Menad, 2012).

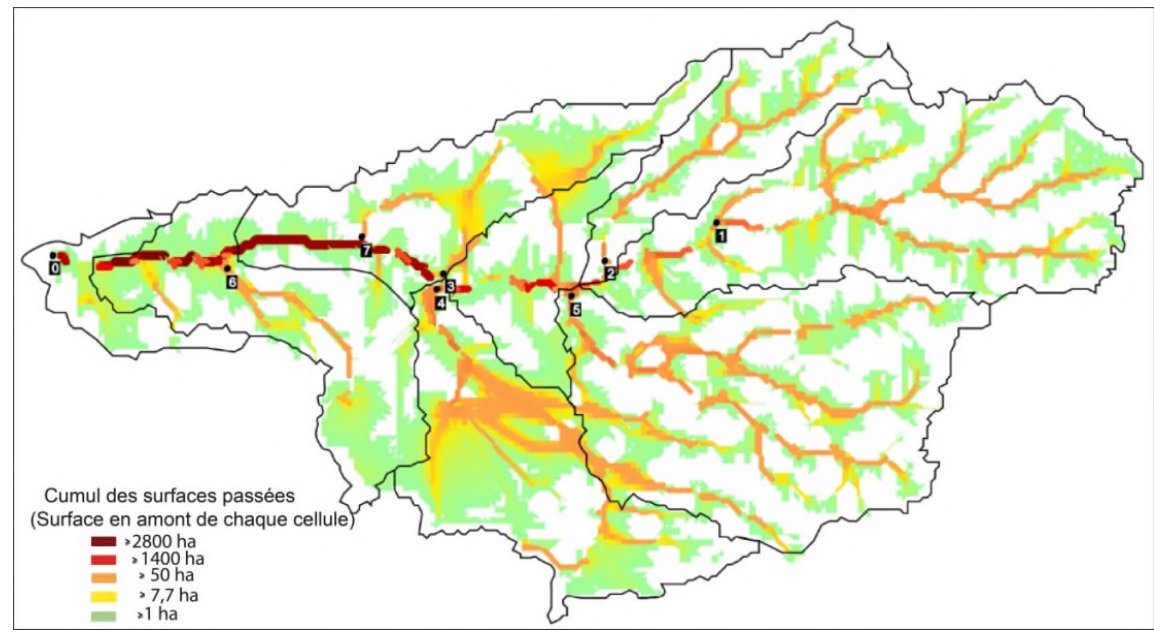

Fig. 4 : Cartographie de la réponse surfacique des différentes sous-unités hydrographiques de l'Oued Koriche, (modifier selon Menad, 2012). 

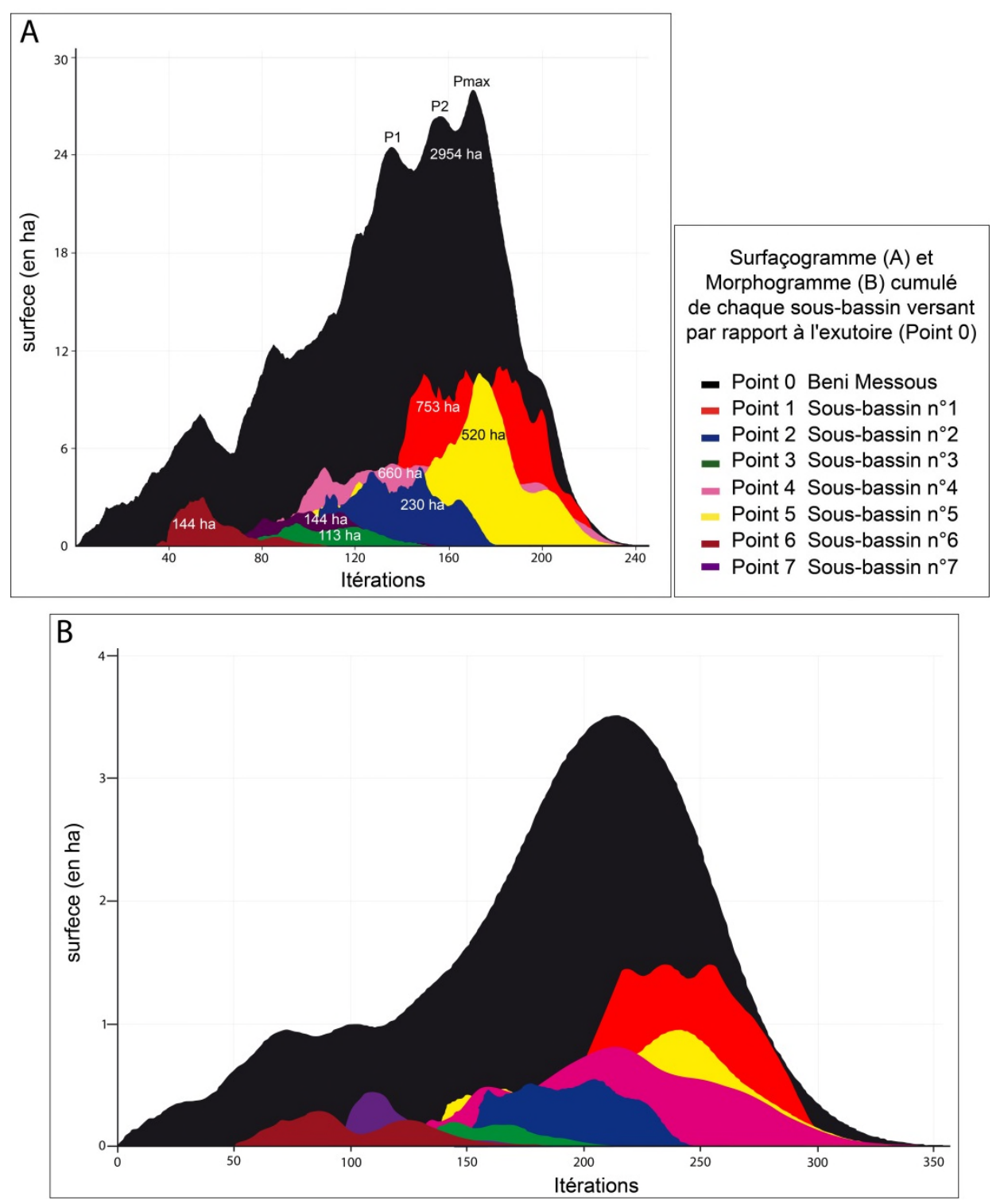

Fig. 5 : A : Surfaçogramme ; B : Morphogramme de la réponse surfacique des différentes sous-unités hydrographiques de l'Oued Koriche en utilisant l'automate RuiCells.

\section{V.2. Impact de l'anthropisation sur l'évolution spatio-temporelle de la réponse hydrologique}

Aux différents inputs implémentés dans un premier temps (facteurs météorologique et hydro-morphologique) on intègre les cartes de vulnérabilité établies auparavant (cf. supra), afin de qualifier (facteur aggravant ou pas) et quantifier les impacts de l'anthropisation. Il est nécessaire ici de simuler le comportement hydrologique à des états d'occupation du sol distincts (antérieurs, actuel, futurs), cela permet, à la fois, de comprendre comment ces derniers ont pu modifier, en aggravant ou pas, le risque hydrologique (débits de pointe et les volumes ruisselés), et de prévoir comment les future plans territoriaux vont l'influencer.

Quand il s'agit de quantifier l'effet de l'anthropisation, les caractéristiques de l'événement météorologique (cumuls, intensité, répartition spatio-temporelle) sont des points importants à étudier. Dans certains cas, la magnitude même de l'aléa hydrologique trouve une explication dans la distribution des pluies dans le temps. On constate par exemple que le caractère intense des pluies à tendance de rendre les surfaces rapidement ruisselantes, minimisant ainsi le rôle aggravant joué par l'emprise urbaine (imperméabilisation des sols); les répar- 
titions spatiales des pics de l'événement pluvieux peuvent également forcer le caractère polygénique de la réponse hydrologique (i.e., arrivée concomitante des réponses hydrologique des différents sous-bassins versants).

Les outputs des modèles de simulations globaux, et selon les caractéristiques du territoire, du phénomène hydrologique et du type d'information recherché, vont être complétés par des modèles de simulation spécialisés voire très spécialisés. Qui eux permettent d'effectuer des analyses très poussées et raffinées afin de traiter les anomalies relevées auparavant par les outputs des modèles de simulation globaux.

\section{V.3. Apport de la méthode hydrosystémique}

Elle permet de cerner, le plus possible, le fonctionnement de l'hydrosystème autant qu'entité, et de comprendre ainsi les liens et les interactions existants entre les différents paramètres intervenant dans la genèse et le développement spatio-temporelle de la réponse hydrologique au sein du bassin-versant. Ce cernement global de l'hydrosystème permet une meilleure évaluation du risque.

Par le passé on a pu constater des travaux d'aménagement qui avaient comme but principal, de réduire le risque hydrologique par endroit, et qu'après avoir été mise en place, on a remarqué qu'ils n'ont fait que déplacer voire aggraver le risque à d'autres parties du bassin versant. L'utilisation des outils de simulation numérique va nous éviter en partie ce genre de problème du fait qu'elle nous donne la possibilité de tester des scénarios avant leur réalisation sur le terrain.

\section{VI. Étude du risque hydrologique via une analyse systémique}

Dans le cas où le niveau du risque évalué par les démarches évoquées auparavant (démarche thématique et démarche globaliste) ne reflète pas la hauteur des dégâts observés réellement sur le terrain, il est donc indispensable de pousser l'analyse plus loin. Il faut aller chercher au-delà de la magnitude de l'événement météorologique, des caractéristiques physiographique et des paramètres anthropiques supportés par les modèles de simulation numérique (i.e., le meilleur logiciel de simulation numérique existant actuellement n'atteint la maturité, et ne peut prendre en compte l'ensemble des enjeux physique, économique, social et politique). Pour y parvenir, la réalisation d'une analyse systémique est fortement recommandée (Arnaud-Fassetta et Fort, 2009 ; Arnaud-Fassetta et al., 2009 ; Douvinet et Delahaye, 2010 ; Menad, 2012). Cette dernière permet d'identifier et de hiérarchiser l'ensemble des variables (i.e., facteurs prédisposants, déclenchants et aggravants), naturelles ou anthropiques, qui peuvent expliquer l'ampleur des dégâts observés sur le terrain.

Il est à noter ici, que les deux premiers facteurs de l'analyse systémique, à savoir facteurs prédisposants et déclenchants, ont été étudiés en grande partie au cours des deux premières évaluations (approches thématique et globaliste), donc il s'agit ici de reprendre leurs résultats et de d'effectuer une lecture synthétique de ces derniers.

\section{VI.1. Facteurs prédisposants}

Un nombre de bassins-versants présente une prédisposition naturelle à la formation de certains comportements hydrologiques à risque. Cette prédisposition est qualifiée à travers les facteurs géologiques, formations superficielles, physiographie du bassin-versant, les caractéristiques climatiques.

\section{VI.2. Facteurs déclenchants}

Le déclenchement d'une réponse hydrologique à risque (prise en charge par ce travail), au niveau d'un bassin-versant est souvent le résultat du passage d'un événement météorolo- 
gique à caractère intense. Le facteur déclenchant est étudié à travers la compréhension des mécanismes intervenants dans leur genèse, la reproduction de leurs répartitions temporelle (hyétogramme) et spatiale (localisation de l'épicentre).

\section{VI.3. Facteurs d'aggravation}

Les facteurs aggravants se résument principalement aux facteurs anthropiques. L'anthropisation peut aggraver le risque hydrologique directement en augmentant la densité des installations dans le bassin-versant à considérer (augmentation du niveau de vulnérabilité), ou encore indirectement, à travers toutes interventions humaines modifiant les caractéristiques physiographique et hydrologique du bassin-versant. L'ensemble de ces modifications doit être identifié sur le terrain, puis leurs effets (aggravant ou pas) sur le risque hydrologique encouru vont être évalués.

Pour bien mener cette étape un travail de terrain minutieux doit être engagé :

\section{VI.3.1. Travaux d'artificialisation du réseau hydrographique}

En milieu urbain, le réseau hydrographique est souvent remplacé par un système artificiel de récupération et d'évacuation des eaux pluviales. Afin de comprendre leurs effets, sur la genèse et l'évolution de la réponse hydrologique, un nombre d'informations doit être recueilli, dont on peut citer : la nature des collecteurs (uniques ou mixtes assurant donc l'évacuation à la fois des eaux usées et des eaux pluviales), la structure interne du réseau et le dimensionnement de ses différents tronçons, la localisation et l'analyse des points de changements de sections (ces derniers quand ils sont mal conçus ils peuvent présenter un risque d'endommagement local), localisation et caractérisation des ouvrages hydrauliques de connexion (dimensions, mode de fonctionnement, capacité maximale théorique d'évacuation, son état actuel, mode et planning des travaux d'entretiens, estimation de sa capacité d'évacuation réelle, ...) ; comparaison de la capacité d'évacuation réelle du réseau avec l'évolution des surfaces drainées (donc les volumes générés), etc.

Il est important ici de fournir des informations concernant les futurs projets qui vont modifier la nature et/ou la capacité d'évacuation du réseau. Il faut garder en tête que l'analyse systémique est un document qui sera utilisé pour développer une politique de gestion préventive du risque. Ainsi, de prendre en compte les projets prévus dans un futur proche rend cette dernière cohérente avec la réalité.

\section{VI.3.2. Analyse plus poussée des modes d'occupation du sol}

Les démarches thématiques et les simulations numérique, menées auparavant, ont permis de reproduire et d'analyser l'évolution de d'occupation du sol et certains de leur impact sur la genèse et le développement de la réponse hydrologique. Néanmoins, les modèles de simulation hydrologique ne supportent souvent pas l'ensemble des éléments de l'environnement. Ainsi, un travail plus poussé et minutieux est à entreprendre sur le terrain (échelle du sous-bassin versant, ponctuel).

Dans le cadre de notre méthode, dans un premier temps, on propose de procéder avec, la production d'une cartographie détaillée sur laquelle on superpose le maximum de couches d'informations établies auparavant (limites des sous bassins-versants, réseau hydrographique, classe de pentes, carte d'occupation du sol, réseau d'assainissement, les différents types d'ouvrages hydrauliques : ouvrage de connexion, avaloirs ou tout autre point de pénétration des écoulements ; zones de confluences, bande de cellules équidistantes, ...). La mise en connexion de l'ensemble des éléments cités ci-dessus permet de faire ressortir de nouvelles anomalies et apporte de nouveaux éléments explicatifs concernant le niveau de risque réellement observé sur le terrain. Le travail du terrain consiste à inspecter ces anomalies, ainsi que d'en 
chercher d'autres.

On peut citer certains nombres de points à inspecter lors d'un travail de terrain mené sur des basins-versants urbanisés :

- Niveau d'entretien du réseau hydrographique et des parties aériennes du réseau d'évacuation, car cela peut modifier significativement leur capacité théorique d'évacuation ;

- Localisation des segments du réseau hydrographique enterrés et l'inspection de leur environnement. Pour des événements de fortes intensités qui s'abattront sur des bassinsversants à système de pente efficace, les capacités d'infiltration des eaux, dans le sol et/ou dans le réseau d'évacuation, sont dépassées par le caractère torrentiel des écoulements, ce qui maintient la circulation des écoulements en surface.

- Observer l'orientation des routes principales par rapport au système de pente, une orientation vers la direction de la pente augmentera la vitesse des écoulements et transformera les routes en axes favorables à leur concentration;

- Localisation des ouvrages hydrauliques de connexions (réseau hydrographique au réseau d'assainissement) par apport aux zones de confluence et la bande principale des cellules équidistantes responsable de la production du Qmax.

- Examiner l'efficacité du réseau d'évacuation lors d'un épisode pluvieux, dont on peut observer la capacité d'évacuation réelle, repérer les points de concentration des eaux pour créer de nouveaux avaloirs, repérer également les points d'entrées inefficaces et les supprimer.

- Relever certaines pratiques qui influencent la capacité théorique du système d'évacuation comme le largage des déchets ménagères (décharge sauvage) et des débris de constructions, etc. Tant de pratiques qui à la fois diminuent la capacité d'évacuation et augmentent le taux de recharge des eaux en matières solides. Cela augmente également le risque de formation d'embâcles au niveau des points de connexions au réseau d'évacuation.

\section{VI.3.3. Politique de gestion et de prévention du risque hydrométéorologique}

Lors de la démarche thématique, on a mené une étude législative synthétique en matière de gestion et de prévention du risque, dont on a dressé le contexte local dans lequel se développe le bassin-versant à considérer, puis on l'a restitué dans un contexte plus large (régional, mondial). À ce niveau, et par le biais des enquêtes et des observations faites sur le terrain on va vérifier ou en est réellement dans leurs applications, souligner les difficultés rencontrées dans leur mise en œuvre; vérifier le taux de coordination entre les différents services concernés, et le niveau d'inscription de cette législation dans une politique de gestion durable du risque.

Un même aléa qui frappera deux villes méditerranéennes à vulnérabilité très proche (la vulnérabilité telle qu'elle est définie dans ce travail ; cf. supra) peut s'exprimer avec deux niveaux de risques très distincts. Cette différence s'explique en grande partie par les politiques de gestion adoptées et de leur concrète efficacité sur le terrain.

\section{VI.4. Apport de l'analyse systémique}

À l'état actuelle des connaissances et selon la complexité du terrain étudié (tel le milieu urbain), les modèles de simulation et/ou modélisation, ne peuvent pas supporter tous les facteurs de l'environnement, et le recourt à l'expertise humain pour explorer d'autres champs et chercher des réponses auprès de ces facteurs reste nécessaire. L'analyse systémique ainsi conçue va permettre de réaliser une synthèse des évaluations précédemment menaient puis aller 
chercher et évaluer ces facteurs non supportés par les modèles et qui sont susceptibles d'influencer la genèse et le développement de la réponse hydrologique et donc le niveau du risque.

\section{Cartographie et gestion du risque}

L'absence d'une carte du risque contribue fortement à la propagation d'une culture d'ignorance chez les gestionnaires des collectivités locales et chez les citoyens. La cartographie du risque hydrométéorologique est loin d'être une tâche facile, en particulier en milieu urbain qui est marqué par une anthropisation aux effets souvent irréversibles (artificialisation du réseau hydrographique, modification du système de pente, imperméabilisation des sols, etc ...). Le risque hydrométéorologique s'inscrit dans un système très complexe non seulement par son aléa à plusieurs facettes (événement météorologique, complexité du système hydomorphologique, réponse hydrologique de nature multiple...) mais aussi en raison d'une vulnérabilité à enjeux multiples.

À la lumière de l'ensemble des analyses (thématique et systémique), et des simulations numériques réalisées le long de cette démarche des supports cartographiques illustrant le niveau de l'aléa, le niveau de vulnérabilité et le niveau de risque dans le bassin étudié (i.e. la carte du risque résulte de la confrontation du niveau de l'aléa et de la vulnérabilité ; Fig.6).

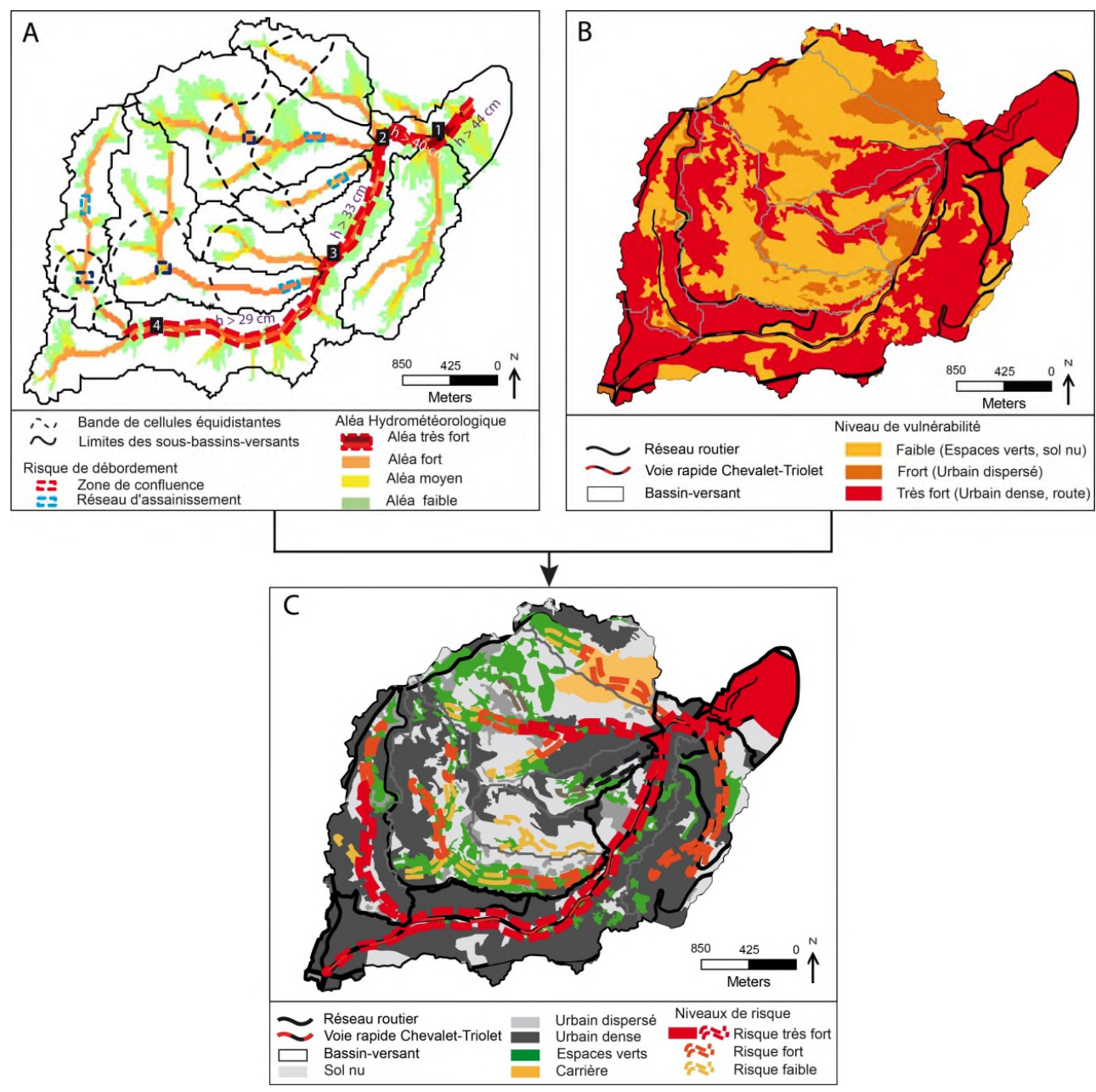

Fig. 6 : Cartographie de risque hydrométéorologique dans le bassin-versant de l'Oued Koriche. A : carte d'aléa. B : carte de vulnérabilité. C: carte de risque,

(modifiée selon Menad, 2012). 


\section{Conclusion}

Dans ce travail on a essayé d'élaborer une démarche d'évaluation globale des risques hydrologique déclenchés par des événements météorologique qui frappent le bassin méditerranéen. Une démarche conçue pour pouvoir être appliquée à des territoires non étudiés auparavant et avec le souci d'être transposable à d'autres terrains à leur image et concourant un même type de risque. Pour y parvenir on s'est mis la contrainte de travailler uniquement avec des données disponibles et facilement accessibles (bases de données internationales ; des données gratuites ou acquises à prix symboliques).

Le développement de démarche globaliste nécessite un travail pluridisciplinaire (science de la terre, hydrologie, sciences sociales et humaines), et l'implication de nombreux outils de traitements (outils de traitement classique, outils de simulation numérique, SIG). Le but étant de pousser le plus loin possible les connaissances liées aux mécanismes de la genèse et de l'évolution temporelle de la réponse hydrologique en tout point du bassin-versant et ainsi assurer une meilleure compréhension de l'hydrosystème et donc une meilleure évaluation puis gestion du risque.

En appliquant cette démarche sur le Massif de Bouzaréah, et en utilisant uniquement des moyens de très faibles coûts, on a pu prendre entièrement en charge le risque hydrologique (identification_analyse et évaluation_cartographie). Cette feuille de route ainsi conçue sert à encadrer et à aider les acteurs, impliqués dans l'évaluation du risque, dans leur travail. Elle démontre également que l'absence de ce genre d'étude, souvent expliquée par le manque de moyen, est en réalité le résultat d'un manque de conscience vis-à-vis de l'importance que présente cette information lors du développement d'une politique de gestion durable du territoire.

\section{Références}

Ambroise B., 2012. Quelques problèmes (et solutions) en modélisation hydrologique. Revue sciences et technique. LJEE $N^{\circ} 20.16$ p. Disponible sur : http://www.ensh.dz/files/ljee/20/1.Article_Ambroise.pdf

Argence S., 2008. Prévisibilité de précipitations intenses en Méditerranée : impact des conditions initiales et application aux inondations d'Alger de novembre 2001. Thèse de doctorat en physique de l'atmosphère, Université Paul Sabatier (Toulouse 3), 160 p.

Arnaud-Fassetta G., Astrade L., Bardou E., Corbonnois J., Delahaye D., Fort M., Gautier E., Jacob N., Peiry J.-L., Piégay H., Penven M.-J., 2009. Fluvial geomorphology and flood-risk management. Géomorphologie : relief, processus, environnement, 2, 109-128.

Arnaud-Fassetta G., Fort M., 2009. The integration of space of good functionment in fluvial geomorphology, as a tool for mitigating flood risk. Application to the left-bank tributaries of the Aude River, Mediterranean France. In Gumiero B., Rinaldi M., Fokkens B. (Eds.) IVth ECRR International Conference on River Restoration 2008. Centro Italiano per la Riqualificazione Fluviale, 313-322.

Barozet, O., Gilard, O., Gleyses, G., Sommier, M., 1994. Le modèle de gestion hydraulique bassin versant de la Bourbre. Congrès de la société hydrotechnique de France Crues et inondations 23èmes journées de l'hydraulique, Nîmes, 14-15-16 septembre 1994. p. 727 - 734.

Barroca B., Mouchel J.-M., Bonierbale T., Hubert G., 2008. Chapter 13: Flood Vulnerability Assessment Tool (FVAT). In (Thévenot D.R. Ed.): DayWater: Adaptive Decision Support System for Integrated Urban Stormwater Control. IWA Publishers, London, 119-127.

Chaboureau J-P., Claud C., Cammas J.P., Mascart P., 2001. Large-scale cloud, precipitation 
and upper- level features during FASTEX as inferred from TOVS observations, J. Geophys. Res., 106 (D15), 17293-17302.

Delahaye D., 2002. Apport de l'analyse spatiale en géomorphologie - modélisation et approche multiscalaire des risques. Mémoire d'Habilitation à Diriger des Recherches, 2 tomes, Université de Rouen, Laboratoire Modélisation et Traitements Graphiques (MTG), 250 p.

Delrieu G (Dir)., 2005. Observatoire Hydro-météorologique Méditerranéen ; CévennesVivarais. Rapport éta-blie dans le cadre de l'évaluation/labellisation des dispositifs d'observation de l'environnement par le Comi-té Inter-Organisme, p. 26. Disponible sur : http://www.cnrm.meteo.fr/cyprim/global/documents_chantier _mediterranee/extr_dossier_ORE.pdf.

Douvinet J., 2010. Le traitement des inondations en catastrophe naturelle : un système à adapter. Risques - Les cahiers de l'assurance, $\mathrm{n}^{\circ} 83,104-112$.

Douvinet J., Delahaye D., 2010. Caractéristiques des «crues rapides » du nord de la France (Bassin parisien) et risques associés. Géomorphologie : relief, processus, environnement, $\mathrm{n}^{\circ} 1$, 73-90.

Douvinet J., Delahaye D., Langlois P., 2008. Modélisation de la dynamique hydrologique potentielle d'un bassin versant et mesure de son efficacité structurelle. Cybergéo, 412.

Ducrocq V., Nuissier O., Ricard D., Lebeaupin C., Thouvenin T., 2007. A numerical study of three catastrophic events over Southern France. Part II: Mesoscale triggering and stationarity factors. Q. J. R. Meteorol. Soc. 134, 131-145.

El Saidi M., Mellas S., J. Douvinet., Nasrallah W., Fehri N., Menad W., Vinet F. (2016) Chapter: Urbanization and land use as a driver of flood risk. In book: The mediterranen region under climate change, Edition: INSTITUT DE RECHERCHE POUR LE DÉVELOPPEMENT Marseille, Publisher: AllEnvi, Editors: Stéphanie Thiébault, Jean-Paul Moatti, pp.563 $-575$

Ghidaglia J-M et Rittaud B, 2004. La simulation numérique, La recherche, $\mathrm{n}^{\circ}$ 380, pp 73-76

Hamidi Boudjelthia H., Douba A., (2016) - Les risques hydrologiques dans la wilaya de Chlef (évaluation et cartographie). Mémoire de fin d'études présenté pour l'obtention du diplôme d'ingénieur en master en hydraulique (option : hydraulique urbaine). Université de Hassiba Benbouali, $100 \mathrm{p}$.

Harvey D.P., 2002. A Generic Modelling Framework Componcent for Htdroinformatics Systems. PhD thesis, University of Bridtol, Bristol, U.K. xix, 193 p.

Homar V., Romero R., Stensrud D. J, Ramis C., Alonso S., 2003. Numerical diagnosis of a small, quasi-tropical cyclone over the western Mediterranean : Dynamical vs. boundary factors, Q. J. R. Meteorol. Soc., n ${ }^{\circ} 129,1469-1490$

Howard A.D., 1967. Drainage analysis in geology interpretation: a summation, Bull. Am. Assoc. Petrol. Geol., Tulsa, n51, 2246-2259.

Hubert G et Ledoux B., 1999. Le coût du risque. L'évaluation des impacts socio-économiques des inondations. Presses de l'École Nationale des Ponts et Chaussées, 20-83.

Langlois P., Delahaye D., 2002. Ruicells, un automate cellulaire pour la simulation du ruissellement de surface. Revue Internationale de Géomatique, 12-4, 461-487. 
Lasat M-C et Rodriguez R., 1997. Towards a regionalization of extreme rainfall events in the Mediterranean area. In TRIEND'97; Regional Hydrology : Concepts and Models for Sustainable Water Resource Manage-ment.. IAHS Press. Wallingford, Oxfordshire, n²46, 215-222.

Lebeaupin C., Ducrocq V., Giordani H., 2006. Sensitivity of torrential rain events to the sea surface temperature based on highresolution numerical forecasts. J. Geophys. Res. n 111. $19 \mathrm{p}$.

Maheras P., Flocas H-A., Patrikas I., Anagnostopoulou C., 2001. A 40 year objective climatology of surface cyclones in the Mediterranean region: spatial and temporal distribution. International Journal of Climatolo-gy, $\mathrm{n}^{\circ} 21,109-130$.

Menad W. (2005) - Caractérisation des zones inondables dans la Wilaya de Chlef : Etat actuel et évaluation. Mémoire de fin d'études présenté pour l'obtention du diplôme d'ingénieur en Biologie spécialité Ecologie et Environnement, Université de Hassiba Benbouali, 90 p.

Menad W. (2012) - Risques de crue et de ruissellement superficiel en métropole méditerranéenne : cas de la partie ouest du Grand Alger. Thèse de doctorat en Dynamique des milieux et risques, Université de Paris Diderot (Paris 7), 330 p.

Menad W., Douvinet J., Beltrando G., Arnaud-Fassetta G., 2012. Evaluer l'influence de l'urbanisation face à un aléa météorologique remarquable : les inondations des 9-10 novembre 2001 à Bab-el-Oued (Alger, Algérie). Vol. 03. Géomorphologie : relief, processus, environnement, sous presse, 81-94

Peguy Ch.P., 1942. Principes de morphométries alpine. Revue Géographique Alpine, 2, pp. 453-486.

Shreve R.L., 1966. Statistical law of stream numbers. Journal of Geology, n74, 17-37.

Torterotot J. P., 1993. Le coût des dommages dus aux inondations : Estimation et analyse des incertitudes, Thèse de doctorat, spécialité Sciences et Techniques de l'Environnement, Ecole Nationale des Ponts et Chaussées, 284 p. 